\title{
植物器官脱落分子生物学研究进展
}

\author{
王翔 陈晓博 ${ }^{* *}$ 李爱丽 毛 龙 ${ }^{*}$ \\ 中国农业科学院作物科学研究所 / 国家基因资源与遗传改良重大科学工程, 北京 100081
}

摘 要: 植物器官脱落(organ abscission)是自然界普遍的现象。器官脱落发生的区域叫做离区(abscission zone)。器官 脱落时离区细胞的细胞间质和细胞壁发生降解, 导致远端器官离开母体。离区的发育和功能行使是多种基因参与的 精确而复杂的调控过程。落粒性是作物栽培和育种中的重要农艺性状，是植物器官脱落的典型形式之一。落粒性适 宜的作物品系驯化是人类文明史上最重要的成就之一，但直到近年人们才对禾本科植物落粒的分子机制有了新的认 识。本文重点综述拟南芥、水稻、番茄等模式植物中离区发育和器官脱落的分子生物学研究进展, 并对今后的研究 方向做了简要展望。

关键词：离区; 器官脱落; 落粒性

\section{Advances in Molecular Biology Study of Plant Organ Abscission}

\author{
WANG Xiang, CHEN Xiao-Bo ${ }^{* *}$, LI Ai-Li, and MAO Long ${ }^{*}$ \\ Institute of Crop Sciences, Chinese Academy of Agricultural Sciences / National Key Facility for Crop Gene Resources and Genetic Improvement, \\ Beijing 100081, China
}

\begin{abstract}
Organ abscission is a ubiquitous phenomenon in the plant kingdom. Abscission occurs at specific sites called abscission zones (AZs). The development and functioning of AZs are complex and precise processes which involve many genes. Degradation of cell wall and middle lamella at AZs causes cell separation and leads to shedding of distal organs. Seed-shattering is one of the most important traits in crop cultivation and breeding and is a typical case of organ shedding. Domestication of non-shattering crop lines is one of the greatest achievements of human civilization, but untill recently its molecular mechanisms started to be revealed. This review focuses on the advances in the molecular biology study of abscission zone development and organ shedding in Arabidopsis, rice, and tomato. The paper provides a brief perspective for the future research in this important arena.
\end{abstract}

Keywords: Abscission zone; Organ abscission; Seed-shattering

植物器官脱落不仅对于物种的繁殖和传播有重 要的生物学意义, 而且对于农业生产具有重要的影 响。在长期作物驯化实践中, 人类不断地对作物器 官脱落特性加以选择, 成功地解决了禾本科植物落 粒、断穗, 棉花落铃, 豆类作物提前开荚等问题, 减 少了产量损失。在番茄中, 无离区品种的应用不仅 可以提高机械收获和后续加工的效率, 而且可以减 少果柄对果实造成的机械损伤, 提高果品质量。对 于植物器官脱落的研究, 早期主要侧重于生理生化 方面。近年来随着分子遗传学和细胞生物学的发展, 解析离区发育和器官脱落的分子机制成为分子生物 学研究的热点之一。

\section{1 器官脱落及其原因}

器官脱落(organ abscission)是指植物组织或器 官脱离母体的生理过程。它是植物应对外界环境(如 病原物侵染)或放弃那些不再发挥作用的器官的有 效机制。器官脱落的组织区域及邻近的数层细胞被 称为离区 (abscission zone, AZ $)^{[1]}$ 。器官脱落可以是衰 老或成熟引起的, 比如果实、种子的脱落; 也可以是 自身生理过程引起的, 例如营养生长和生殖生长的 竞争引起的落花等; 逆境条件如干旱、高温、病虫 害等是其另一主要原因。

激素, 尤其是乙烯在植物器官脱落过程中起着 重要的作用 ${ }^{[2-3]}$ 。但一些植物(如柑橘)的果柄并不受

\footnotetext{
本研究由国家自然基金资助项目(30670188)资助。

*通讯作者(Corresponding author): 毛龙, E-mail: maolong@caas.net.cn; Tel: 010-82105861 ${ }^{* *}$ 共同第一作者 Received(收稿日期): 2008-08-11; Accepted(接受日期): 2008-10-24.
} 
乙烯诱导而脱落 ${ }^{[4]}$ 。生长素在器官脱落中与乙烯的 作用相拮抗，它控制离区细胞在适当的时间对乙烯 产生应答 ${ }^{[5]}$ 。ABA (abscisic acid), 即脱落酸, 对一些 植物的叶片和花器官脱落具有诱导作用, 但有实验 表明其作用是通过乙烯间接实现的 ${ }^{[6]}$ 。植物器官的 脱落是多种激素平衡和协同作用的结果。脱落过程 往往伴随一系列基因表达的变化, 特别是编码细胞 壁降解酶基因如 1,4- $\beta$-葡聚糖酶、多聚半乳糖醛酸酶 等的上调表达。另外, 扩展蛋白(expansin)、病程相 关蛋白 (pathogenesis-related proteins) 以及其他一些 蛋白在器官脱落过程中也起着重要的作用 ${ }^{[5]}$ 。

\section{2 拟南芥离区发育相关基因}

拟南芥中存在两种与离区发育和器官脱落相关 的生物过程, 即花受粉后花墓、花瓣、雄蕊等花器 的脱落过程和果莱开裂过程。这些生物过程的存在 使拟南芥成为研究离区发育和器官脱落的理想模 式。目前, 通过篩选突变体, 已鉴定出多个与拟南芥 花离区发育和器官脱落相关的基因(表 1), 从而初步 建立了拟南芥花器官脱落的调控网络。

拟南芥中的 $B O P 1$ (blade on petiole1)和 $B O P 2$ 是 控制花器官离区发育的一对功能重复的基因。BOP1 和 BOP2 基因各自分别编码一个叫做病程相关基因 非表达子 1 (nonexpressor of pathogenesis-related genes
1)的转录因子。在双突变体 bop1bop2 中, 花器官离 区不能形成, 其他性状如叶柄发育和器官的非对称 性生长等也受到不同程度的影响 ${ }^{[7]}$ 。BOP 基因抑制 KNOX (knotted1 like homeobox)基因(决定分生组织特 异性) KNAT1 (knotted-like from Arabidopsis thaliana)/ $B P$ (bervipedicellus)、KNAT2 和 KNAT6 在叶中表达。 而基因敲除实验证明 $K N A T / B P$ 影响拟南芥花器官的 离区发育。如在 $b p$ 突变体中, 花器官离区形成更多 的泡状细胞, 使之提前脱落 ${ }^{[8]}$ 。

在拟南芥中，尽管大多数影响花器官脱落的基 因都不同程度地提前或延迟脱落过程，但是这些基 因都不影响离区的形成，如拟南芥突变体 ida (inflorescence deficient in abscission)花器官的离区 发育正常, 但即使在种子成熟后其花器官也不能正 常脱落。IDA 只编码 77 个氨基酸残基, 还有高的 $\mathrm{p} I$ (等电点)值, 且 $N$ 端具有疏水性。因此, IDA 可能是 一个新的类似于 CLV3 (clavata 3)的配体, 为一类可溶 性蛋白, 能够与其受体结合启动下游的信号传递 ${ }^{[9]}$ 。 过量表达 IDA 的拟南芥株系表现出花器官的提前脱 落, 而且花柄、茎生叶、花序等都自基部提前脱落, 果荚提前开裂，但种子脱落并不受影响。所有实验 均表明 IDA 是一个脱落的正调控因子 ${ }^{[10]}$ 。随后的实 验结果显示, IDA 蛋白 C 端叫做 EPIP 的 21 个保守肽 段就完全可以恢复突变体 ida 的表型, 表明这个保守

表 1 影响拟南芥花离区发育和花器官脱落的基因

Table 1 Genes involved in AZ formation and organ shedding in Arabidopsis

\begin{tabular}{|c|c|c|c|}
\hline $\begin{array}{l}\text { 基因名称 } \\
\text { Gene name }\end{array}$ & $\begin{array}{c}\text { 类型 } \\
\text { Gene type }\end{array}$ & $\begin{array}{c}\text { 功能 } \\
\text { Function }\end{array}$ & $\begin{array}{c}\text { 文献 } \\
\text { Reference }\end{array}$ \\
\hline BOP1/2 & $\begin{array}{l}\text { 病程相关非表达子 } 1 \\
\text { Nonexpressor of } P R \text { genes (NPR1) }\end{array}$ & $\begin{array}{l}\text { 决定花器官离区分化 } \\
\text { Specify AZ differentiation of flower organ }\end{array}$ & McKim et al. ${ }^{[7]}$ \\
\hline KNAT1/BP & $\begin{array}{l}\text { Knox 基因 } \\
\text { Knox gene }\end{array}$ & $\begin{array}{l}\text { 影响离区发育 } \\
\text { Affect development of AZ }\end{array}$ & Wang et al. ${ }^{[8]}$ \\
\hline$I D A$ & $\begin{array}{l}\text { 分泌型蛋白 } \\
\text { Secreted small protein }\end{array}$ & $\begin{array}{l}\text { 正调控花器官脱落 } \\
\text { Positively regulate shedding of flower organ }\end{array}$ & Butenko et al. ${ }^{[9]}$ \\
\hline HAESA/HSL2 & $\begin{array}{l}\text { 类受体蛋白激酶 } \\
\text { Receptor-like protein kinase }\end{array}$ & $\begin{array}{l}\text { 控制花器官脱落 } \\
\text { Regulate shedding of flower organ }\end{array}$ & $\begin{array}{l}\text { Jinn et al. } .^{[12]} \\
\text { Cho et al. }{ }^{[13]}\end{array}$ \\
\hline$M K K 4 / 5$ & $\begin{array}{l}\text { 丝裂原蛋白激酶激酶 } \\
\text { MAPK kinase }\end{array}$ & $\begin{array}{l}\text { 控制花器官脱落 } \\
\text { Regulate shedding of flower organ }\end{array}$ & Cho et al. ${ }^{[13]}$ \\
\hline MPK3/6 & $\begin{array}{l}\text { 丝裂原蛋白激酶 } \\
\text { Mitogen activated protein kinase }\end{array}$ & $\begin{array}{l}\text { 控制花器官脱落 } \\
\text { Regulate shedding of flower organ }\end{array}$ & Cho et al. ${ }^{[13]}$ \\
\hline AtZFP2 & $\begin{array}{l}\text { 锌指蛋白 } \\
\text { Zinc finger protein }\end{array}$ & $\begin{array}{l}\text { 过表达延迟花器官脱落 } \\
\text { Delay shedding of flower organ in over-expression line }\end{array}$ & Cho et al. ${ }^{[13]}$ \\
\hline ARP4/ARP7 & $\begin{array}{l}\text { 肌动蛋白相关蛋白 } \\
\text { Actin-related protein }\end{array}$ & $\begin{array}{l}\text { 突变体延迟花器官脱落 } \\
\text { Delay shedding of flower organ in mutant }\end{array}$ & Kandasamy et al. ${ }^{[17]}$ \\
\hline AGL15 & $\begin{array}{l}\text { MADS 盒基因 } \\
\text { MADS-box gene }\end{array}$ & $\begin{array}{l}\text { 过表达延迟花器官脱落 } \\
\text { Delay shedding of flower organ in over-expression line }\end{array}$ & Fernandez et al. ${ }^{[14]}$ \\
\hline AGL18 & $\begin{array}{l}\text { MADS 盒基因 } \\
\text { MADS-box gene }\end{array}$ & $\begin{array}{l}\text { 过表达延迟花器官脱落 } \\
\text { Delay shedding of flower organ in over-expression line }\end{array}$ & Adamczyk et al. ${ }^{[15]}$ \\
\hline
\end{tabular}


氨基酸序列在植物发育过程中具有重要功能 ${ }^{[1]}$ 。

拟南芥中一个富含亮氨酸重复序列的受体激酶 HAESA, 在花器官脱落中起着重要作用。在 HAESA 的反义抑制转基因植株中, 明显推迟花器官的外 3 轮器官脱落, 而在表型极明显的转基因株系中, 花 器官不会脱落 ${ }^{[12]}$ 。Stenvik 等 ${ }^{[11]}$ 证明 IDA 所产生的 多肽正是通过 HAESA 这个受体来引发拟南芥花器 官的脱落。最近拟南芥花器官脱落的信号途径得到了 更进一步的深入研究, 实验证明 HAESA 下游的 MKK4 (mitogen-activated protein kinase kinase 4)/ MKK5 通 过激活 MPK6 (mitogen-activated protein kinase 6)/ $M P K 3$ 来诱导拟南芥的花器官脱落, 此结果表明是 IDA、HAESA 和 HSL2 (haesa-like2)、以及 MAPK 级联 信号途径的依次作用控制拟南芥花器官的脱落 ${ }^{[13]}$ 。

Patterson 等 $^{[4]}$ 筛选到 5 个花器官延迟脱落突变 体, 命名为 $D A B$ (delayed floral organ abscission)。随 后, 发现这 5 个突变体代表 3 个独立的位点, dab1、 $d a b 3$ 是隐性遗传控制, 而 $d a b 2$ 由显性基因控制, 这 3 个突变体都不同程度地延迟拟南芥的花器官脱落。 转录因子 AGL15 和 AGL18 也影响花器官脱落, 其过 表达推迟花器官脱落, 同时延迟拟南芥的衰老过程 [14-15]。在利用基因芯片分析拟南芥雄蕊离区特异转 录谱实验中, Cai 等 ${ }^{[16]}$ 分离到一个影响花器官脱落的 基因 AtZFP2 (zinc finger protein2), 其过量表达不仅 延迟花器官脱落, 而且显著改变花的形态和育性。 另外, 拟南芥的 $A R P$ (actin-related proteins)家族, 在 花器官脱落过程中也起作用, 利用 RNAi 技术产生 的 ARP7 敲除转基因株系的花器官脱落明显推迟, 而离区发育与野生型的拟南芥没有区别, 并且此转 基因株系对乙烯的三重反应与野生型也一致，表明 $A R P 7$ 控制花器官脱落是独立于乙烯的另一条途径。 类似于 AGL15 和 HAESA, ARP4 在推迟花器官脱落 过程中也有相同的作用 ${ }^{[17]}$ 。

乙烯加速器官脱落而生长素阻止脱落已被人们 广泛接受, 对于拟南芥中激素信号途径缺陷突变体 的鉴定, 有助于剖析激素在花器官脱落中的作用。 乙烯不敏感型突变体 etr1 (ethylene receptor 1)和 ein2 (ethylene-insensitive 2) 都延迟了花器官的脱落, 但 并不能阻止脱落的发生 ${ }^{[12]}$ 。此外, 在拟南芥中, 突变 体 arf2 (auxin response factor 2) 推迟花器官脱落, 而 $A R F 1$ 的突变能够增强 $\operatorname{arf2}$ 的表型。同 ARF1 的作用 类似, NPH4/ARF7 和 ARF19 的突变也能加剧 arf2 花 器官脱落延迟的表型。这些影响生长素合成途径的
转录因子可能通过控制花器官激素的梯度变化来影 响衰老过程和器官脱落过程 ${ }^{[18-19]}$ 。影响乙烯和生长 素合成和信号途径的两组基因通过未知的机制共同 影响拟南芥花器官的脱落过程。

此外, 还有一些影响拟南芥花器官脱落的基因 被发现。遗传研究表明, 一个编码 F-box 蛋白的 HAWAIIAN SKIRT 基因也影响拟南芥花器官的脱落, 但是它的作用并不是直接的, 而是突变体花赏中下 部部分融合使之不能直接脱落 ${ }^{[20]}$ 。

拟南芥的果荚开裂也涉及到类似于器官脱落的 过程。在此过程中, 两个重复功能的 MADS-box 基 因 SHP $1 / 2$ (shatterproof $1 / 2$ )控制果荚离层的分化, 而 另外一个 MADS-box 基因 FUL (fruitfull)是果荚发育 的决定基因, FUL 通过负调控 SHP 来共同平衡拟南 芥果莱的正常发育 ${ }^{[21]}$ 。而在随后的实验中，人们发 现更多的基因参与拟南芥果荚离层(separation layer) 的发育过程, 如图 1 所示, FUL 和 RPL 共同作用, 限 制离层发育的 4 个关键基因 SH1、SH2、ALC (alcatraz) 和 $I N D$ (indehiscent) 只在果荚离层表达。FUL 抑制这 4 个基因在果荚中表达(虚线表示间接作用), 而 $R P L$ (replumless)主要限制 $S H 1 / 2$ 以及 $A L C$ 和 $I N D$ 在假隔 膜(replum)中表达。最近又有新一层次的果莱离层发 育的调控被鉴定, FIL(filamentous flower)、YAB3 (yabby3)和 $J A G$ (jagged) 3 个基因在 $F U L$ 和 $S H P 、 A L C$ 和 IND 的上游共同促进这几个基因的表达，而 FIL、 $Y A B 3$ 和 $J A G$ 又受 $R P L$ 的反馈抑制。AS (asymmetirc leaves)在果荚中高水平表达从而抑制 $K N A T 1 / B P$ 基 因, 而在假隔膜中低水平表达从而减弱对 $K N A T 1 / B P$ 基因和 $R P L$ 的抑制, $K N A T 1 / B P$ 基因通过调节 $R P L$ 基因促进假隔膜的发育。果荚的 3 个区域(果荚, 果 荚离层, 假隔膜)由相互拮抗的两组因子形成, 即果 荚决定因子(椭圆形)和假隔膜因子(六边形)。果荚离 层(长方形)形成于果荚决定基因和假隔膜分化基因 相互重叠的区域 ${ }^{[22]}$ 。上述因子形成一个复杂的调控 网络来控制果荚离层发育(图 1$)^{[23]}$ 。Wu 等 ${ }^{[24]}$ 鉴定了 在拟南芥果荚离层中由 7 层细胞组成的区域, 显微 观察表明 $\beta$-葡萄糖醛酸酶在这 7 层细胞中高度表达, 而这 7 层细胞是在花期 15 阶段由细胞的不对称分裂 形成。研究发现 IND 控制果荚离层的 7 层细胞的不 均等分裂。从目前看来这个网络并不完整，还需要 加入更多的因子。在拟南芥中, 还有一个 MADS-box 基因 STK (seedstick)影响种子离区发育从而控制种 子脱落 ${ }^{[25]}$ 。 


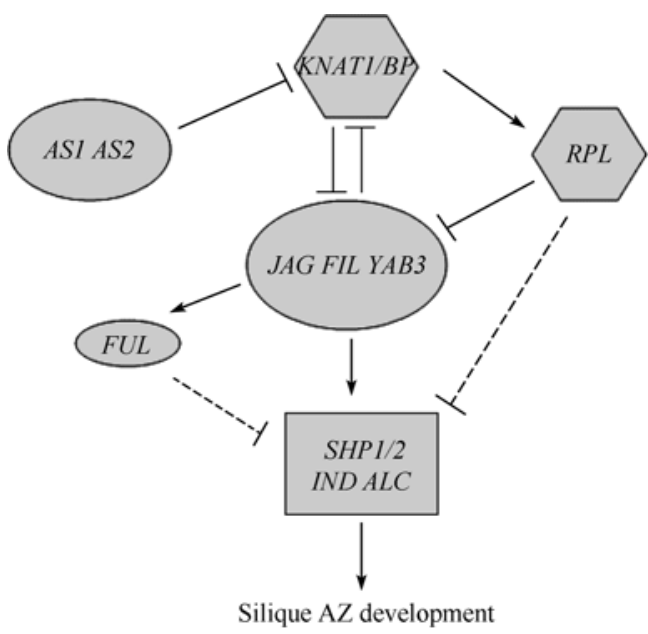

图 1 拟南芥果荚离层发育的网络调控模式图

Fig. 1 Gene regulation network for Arabidopsis silique AZ development

\section{3 水稻离区发育基因}

禾本科植物包含世界上最重要的粮食作物, 如 水稻、小麦等。作物的驯化过程发生于过去大约一 万年的时间内。从野生种中驯化选择不易发生种子 脱落(seed-shattering)的品种类型是人类文明史上最 大的成就之一, 是现代高产品种的最基本要求。在 这漫长的驯化过程中, 人们有意识无意识选择了控 制理想谷粒脱落程度的等位基因, 但直到近几年人 们才对其分子机制的认识有了突破性进展。

2006 年, $\mathrm{Li}$ 等 ${ }^{[26]}$ 利用籼稻品种和一年生野生稻 作亲本得到 $\mathrm{F}_{2}$ 群体, 对水稻中的落粒性状进行 QTL 遗传分析, 发现了一个贡献率为 $69 \%$ 的主效 QTL 位 点, 并且为显性遗传, 命名为 $S H 4$, 最终将其定位到 水稻第 4 染色体的 $1.7 \mathrm{~kb}$ 范围内。这个基因编码一 个未知功能的转录因子, 控制水稻的离区发育。随 后的亚细胞定位结果(定位在细胞核中)也支持 $\mathrm{SH} 4$ 是一个转录因子。转基因实验表明栽培种与野生型 的水稻落粒性只因第 1 个外显子的单核苷酸改变而 使栽培种中的赖氨酸代替了野生种中的天冬氨酸。 人类历史上对落粒性潜意识的选择也正是对这个位 点的选择使水稻不再容易落粒。在水稻中另外一个 与落粒有关的基因 SHA1(shattering1)编码的氨基酸 同 SH4 的氨基酸有 $98 \%$ 序列一致性, 而且是等位基 因。但两者的不同之处在于, SHA1 并不影响离区的 形成 ${ }^{[27]}$ 。

同年, Konishi 等 ${ }^{[28]}$ 采用类似的方法，用易落粒 的 kasalath 和不易落粒的 nipponbare 的 $\mathrm{F}_{2}$ 代群体定 位了一个贡献率为 $68.6 \%$ 的主效 QTL 位点, 该基因
位于第 1 染色体长臂, 编码 BEL1 类蛋白。互补实验 证明单个 $Q S H 1$ 就可以影响离区的发育。随后研究 发现 QSH1 是由 SNP(single nucleotide polymorphism) 控制的顺式作用元件突变引起的，在突变的碱基区 域是一个典型的 $\mathrm{AB} 13$ 类转录因子结合的 RY 重复 区。正是它的突变引起下游一个编码蛋白(replumless 的直系同源基因)表达的改变而引起落粒性状变化, 而此蛋白在两个作图亲本的序列完全一致。随后, Ji 等 ${ }^{[29]}$ 又将另外一个落粒基因 $S H-H$ 定位于第 7 染色 体的两个 SSR 分子标记 RM8262 和 RM7161 之间, 分别相距 $1.6 \mathrm{cM}$ 和 $2.0 \mathrm{cM}$ 。水稻中另外一个影响落 粒的基因是 SPR3, 该基因还影响水稻的穗型, 该基 因突变后水稻散穗，而且容易落粒。同源比对表明 该基因并没有序列相似的已知功能基因, 因此是一 个新的遗传因子 ${ }^{[30]}$ 。

\section{4 番茄离区发育相关基因}

番茄花柄离区是一个位于花柄中部类似关节的 显著结构, 番茄以其独特的花柄结构成为研究离区 发育的又一理想植物。番茄离区发育控制基因 $J$ (jointless)是一个 MADS-box 基因, 它的突变引起番 茄果(花)柄离区的消失。遗传分析发现 $J$ 突变是由于 第 1 个外显子的部分序列连同起始密码子的上游共 $939 \mathrm{bp}$ 的碱基缺失引起的。转基因互补实验证实番 茄果实离区正是由这个基因控制 ${ }^{[31]}$ 。同时发现, 在 番茄中 $J$ 对于花序分生组织特异性和每个花序分生 组织花原基的保守性也是必需的。在突变体番茄中, 花序发育是无限性, 即生殖生长可以再转化到营养 生长; 而在野生型番茄中, 花序发育是有限性的 ${ }^{[22]}$ 。 这说明控制离区发育基因功能的多样性。在拟南芥 中与 $J$ 序列最相近的基因是 SVP (short vegetative phase), 它们的蛋白一致性为 $69 \%$ 。不同的是, SVP 的功能在拟南芥中是抑制开花，与离区发育并无关 系。但在树木大桉(Eucalyptus grandis)中的同源基因 EgrSVP 影响花序发育, 过量表达 EgrSVP 可以引起 花序由有限性、单花序变为无限性、多花序等表型, 类似于 $J$ 的部分功能 ${ }^{[33]}$ 。

对于 $\mathrm{J}$ 的蛋白结构分析显示, 在其氨基酸序列 C 端有一个类似于 AtZFP、AGL15 和 AGL18 中存在 的 DLSLRL 的氨基酸序列, 即 DTSLKL。在拟南芥 中, 这 3 个基因的过量表达株系都表现花器官脱落延 迟。关于这种现象的具体分子机制并不清楚, 但 $j$ 突变体中离区的形成确实被阻断 ${ }^{[16]}$ 。这些结果表明, 
这一氨基酸序列可能对植物器官的脱落过程起着很 重要的作用。

本实验室利用酵母双杂交系统鉴定与 $\mathrm{J}$ 相互作 用的候选 MADS-box 基因。结果表明, 在拟南芥、 番茄、金鱼草的同源蛋白之间表现出相似的互作模 式，但不同种属之间也有不同; 与 $\mathrm{J}$ 相互作用的 8 个 候选 MADS-box 蛋白中的大多数互作得到了 $\mathrm{BiFC}$ (bimolecular fluorescence complementation)系统的确 认; MADS-box 基因中的 MADS 结构域增强了蛋白 之间相互作用的特异性。所有的这些蛋白在植物开 花时间和花器官发育过程中都发挥着重要作用, 表 明 $\mathrm{J}$ 很可能在花器官的发育中扮演着更为重要的角

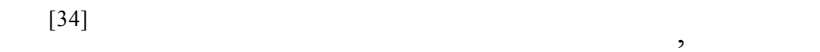
侧组织作为对照, 用 Affymetrix 公司的番茄基因芯 片进行转录谱分析。结果显示, 番茄离区特异上调 表达的基因 WUS (wuschel)、BL (blind)和 LS (lateral suppressor)等均与番茄花序分生组织发育密切相关。 番茄茎尖分生组织的最内层 (L3 层)决定番茄的离区 发育 ${ }^{[35]}$ 。而 WUS 正是在 L3 层高度表达, 与我们的 芯片结果显示一致, 表明 WUS 似乎与番茄花柄的离 区发育密切相关。

另外一个控制番茄离区发育的基因 J2 (jointless 2)最近被定位在第 12 染色体近着丝粒部位, 并初步 确定此基因的候选基因为 ToCPL1 (c-terminal phosphatase-like gene), 它编码的蛋白有 4 个结构域, 即 CTD 类磷酸酶催化区、NL1 互作区、BRCA1 的 C 区和 TFIIF 互作 CTD 磷酸酶区 ${ }^{[36]}$ 。J2 除了影响离区 发育外, 另外一个表型就是花序增生。J2 与 $\mathrm{J}$ 在蛋 白序列和结构上都没有任何相似性, 而且 $\mathrm{J} 2$ 的 C 端 不存在 DLSLRL 类似序列。第 3 个影响番茄花柄离 区发育的基因 $L S$ 编码一个 VHIID 蛋白, $L S$ 基因突变 后, 部分花柄离区发育受到影响。同时发现在 $l s$ 突 变体中, 大多数腋芽的分生组织不会形成, 花中缺 少花瓣 ${ }^{[37]}$ 。所以, 在番茄中影响离区发育基因的作 用都不是单一的, 均不同程度地影响其他性状如花 序发育。由此我们可以推断, 番茄离区发育可能并 不是一个独立的过程, 而是花序分生组织发育中的 一个子单元。

此外, 在番茄中, 还有其他的一些基因本身并 不影响离区的形成, 但确实在器官脱落中起着一定 的作用。在影响植物器官脱落的酶中, 多聚半乳糖 醛酸酶(polygalacturonase)和葡聚糖酶 (glucanase) 是 最重要的两种酶, 它们分别降解细胞壁中的果胶和
纤维素。利用 VIGS (virus-induced gene silencing)系 统特异沉默 TAPGs (tomato abscission-related polygalacturonases)基因, 能够延迟乙烯处理后番茄叶的脱落 和增加叶片所需的断裂力, 表明此类基因在番茄叶 脱落过程中的重要作用 ${ }^{[38]}$ 。番茄 $1,4-\beta$-葡聚糖酶 (cel2)反义抑制转基因植株中, 明显增加了番茄果实 离区的断裂力 ${ }^{[39]}$ 。番茄中的 $C E L 1$ (cellulase1)基因也 参与番茄花脱落过程 ${ }^{[40]}$ 。 del Campillo 等 ${ }^{[41]}$ 从乙烯 处理的番茄中分离到 6 个纤维素酶(cellulase), 它们 在番茄花器官的脱落过程中有不同的表达模式，表 明器官脱落是一个涉及不同纤维素酶激活和抑制的 复杂的多步骤过程, 这些纤维素酶在脱落过程中的 相对重要性受脱落的生理条件所决定。

\section{5 研究趋势和展望}

落粒性是作物栽培和育种中最重要的农艺性状 之一。控制种子和果实的脱落程度是植物器官脱落 分子生物学基础研究在农业实践中的应用。近年来 关于离区发育过程中的信号转导途径和分子机制研 究提出了许多有趣但目前无法解释的科学问题。如 在拟南芥中, 花器官脱落信号传递可能存在乙烯依 赖和非依赖两条途径, 但这两种途径相互交叉、部 分重叠的细节并不清楚。同样, 水稻谷粒和番茄果 实离区发育的基因网络也有待进一步研究。此外, 有关离区发育和器官脱落的研究也应当扩大到其他 重要的粮食和经济作物中, 如棉花的落铃和大豆的 开荚等实际问题。在棉铃脱落方面, 以前的研究主 要集中在植物器官中的激素含量和棉铃脱落的关系 以及脱落过程中发生的各种细胞壁降解酶的活性及 作用，而在该领域的分子机制几乎为空白。随着中 美两国科学家联合开展的“国际棉花基因组测序计 划”的实施及完成，人类有望在不久的将来会对该 领域的分子生物学研究有所突破 ${ }^{[42-44]}$ 。大豆开荚的 研究中也存在着类似的问题, 分子机制方面的研究 还没有突破。在小麦中, 对人们早已观察到的西藏 半野生小麦成熟期下穗轴脱落现象的研究, 可以为 改良现代小麦收获提供理想的品系。小麦由于存在 基因组大、多倍性等阻碍基因克隆的问题，希望能 随着小麦基因组测序的进行和完成得到缓解 ${ }^{[45-47]}$, 从而加快落粒相关基因的克隆速度。

春华秋实, 植物器官脱落在自然界的普遍存在 说明它是一个非常重要的生理过程。器官脱落几乎 涉及植物生长发育的所有方面, 从种子、果实、叶 
子脱落到果荚开裂等。所以, 植物器官脱落的分子 生物学研究一方面将深化人们对其本质的认识, 另 一方面将为其在农业生产上的应用做出重要的贡献。

\section{References}

[1] Bleecker A B, Patterson S E. Last exit: Senescence, abscission, and meristem arrest in Arabidopsis. Plant Cell, 1997, 9: 1169-1179

[2] Uheda E, Nakamura S. Abscission of Azolla branches induced by ethylene and sodium azide. Plant Cell Physiol, 2000, 41: 1365-1372

[3] Wagstaff C, Chanasut U, Harren F J, Laarhoven L J, Thomas B, Rogers H J, Stead A D. Ethylene and flower longevity in Alstroemeria: Relationship between tepal senescence, abscission and ethylene biosynthesis. J Exp Bot, 2005, 56: 1007-1016

[4] Patterson S E, Bleecker A B. Ethylene-dependent and -independent processes associated with floral organ abscission in Arabidopsis. Plant Physiol, 2004, 134: 194-203

[5] Roberts J A, Elliott K A, Gonzalez-Carranza Z H. Abscission, dehiscence, and other cell separation processes. Annu Rev Plant Biol, 2002, 53: 131-158

[6] Sagee O, Goren R, Riov J. Abscission of citrus leaf explants: Interrelationships of abscisic acid, ethylene, and hydrolytic enzymes. Plant Physiol, 1980, 66: 750-753

[7] McKim S M, Stenvik G E, Butenko M A, Kristiansen W, Cho S K, Hepworth S R, Aalen R B, Haughn G W. The blade-on-petiole genes are essential for abscission zone formation in Arabidopsis. Development, 2008, 135: 1537-1546

[8] Wang X Q, Xu W H, Ma L G, Fu Z M, Deng X W, Li J Y, Wang Y H. Requirement of KNAT1/BP for the development of abscission zones in Arabidopsis thaliana. J Integr Plant Biol, 2006, 48: $15-26$

[9] Butenko M A, Patterson S E, Grini P E, Stenvik G E, Amundsen S S, Mandal A, Aalen R B. Inflorescence deficient in abscission controls floral organ abscission in Arabidopsis and identifies a novel family of putative ligands in plants. Plant Cell, 2003, 15: 2296-2307

[10] Stenvik G E, Butenko M A, Urbanowicz B R, Rose J K, Aalen R B. Overexpression of Inflorescence deficient in abscission activates cell separation in vestigial abscission zones in Arabidopsis. Plant Cell, 2006, 18: 1467-1476

[11] Stenvik G E, Tandstad N M, Guo Y, Shi C L, Kristiansen W, Holmgren A, Clark S E, Aalen R B, Butenko M A. The EPIP peptide of inflorescence deficient in abscission is sufficient to induce abscission in Arabidopsis through the receptor-like kinases HAESA and HAESA-LIKE2. Plant Cell, 2008, 20: 1805-1817

[12] Jinn T L, Stone J M, Walker J C. HAESA, an Arabidopsis leucine-rich repeat receptor kinase, controls floral organ abscission. Genes Dev, 2000, 14: 108-117

[13] Cho S K, Larue C T, Chevalier D, Wang H, Jinn T L, Zhang S, Walker J C. Regulation of floral organ abscission in Arabidopsis thaliana. Proc Natl Acad Sci USA, 2008, 105: 15629-15634
[14] Fernandez D E, Heck G R, Perry S E, Patterson S E, Bleecker A B, Fang S C. The embryo MADS domain factor AGL15 acts postembryonically: Inhibition of perianth senescence and abscission via constitutive expression. Plant Cell, 2000, 12: 183-198

[15] Adamczyk B J, Lehti-Shiu M D, Fernandez D E. The MADS domain factors AGL15 and AGL18 act redundantly as repressors of the floral transition in Arabidopsis. Plant J, 2007, 50: 1007-1019

[16] Cai S, Lashbrook C C. Stamen abscission zone transcriptome profiling reveals new candidates for abscission control: Enhanced retention of floral organs in transgenic plants overexpressing Arabidopsis zinc finger protein 2. Plant Physiol, 2008, 146: 1305-1321

[17] Kandasamy M K, Deal R B, McKinney E C, Meagher R B. Silencing the nuclear actin-related protein AtARP4 in Arabidopsis has multiple effects on plant development, including early flowering and delayed floral senescence. Plant J, 2005, 41: 845-858

[18] Ellis C M, Nagpal P, Young J C, Hagen G, Guilfoyle T J, Reed J W. Auxin response factor 1 and auxin response factor 2 regulate senescence and floral organ abscission in Arabidopsis thaliana. Development, 2005, 132: 4563-4574

[19] Okushima Y, Mitina I, Quach H L, Theologis A. Auxin response factor 2 (ARF2): A pleiotropic developmental regulator. Plant $J$, 2005, 43: 29-46

[20] Gonzalez-Carranza Z H, Rompa U, Peters J L, Bhatt A M, Wagstaff C, Stead A D, Roberts J A. Hawaiian skirt: An F-box gene that regulates organ fusion and growth in Arabidopsis. Plant Physiol, 2007, 144: 1370-1382

[21] Ferrandiz C, Liljegren S J, Yanofsky M F. Negative regulation of the shatterproof genes by fruitfull during Arabidopsis fruit development. Science, 2000, 289: 436-438

[22] Alonso-Cantabrana H, Ripoll J J, Ochando I, Vera A, Ferrandiz C, Martinez-Laborda A. Common regulatory networks in leaf and fruit patterning revealed by mutations in the Arabidopsis asymmetric leaves 1 gene. Development, 2007, 134: 2663-2671

[23] Lewis M W, Leslie M E, Liljegren S J. Plant separation: 50 ways to leave your mother. Curr Opin Plant Biol, 2006, 9: 59-65

[24] Wu H, Mori A, Jiang X, Wang Y, Yang M. The indehiscent protein regulates unequal cell divisions in Arabidopsis fruit. Planta, 2006, 224: 971-979

[25] Favaro R, Pinyopich A, Battaglia R, Kooiker M, Borghi L, Ditta G, Yanofsky M F, Kater M M, Colombo L. MADS-box protein complexes control carpel and ovule development in Arabidopsis. Plant Cell, 2003, 15: 2603-2611

[26] Li C, Zhou A, Sang T. Rice domestication by reducing shattering. Science, 2006, 311: 1936-1939

[27] Lin Z, Griffith M E, Li X, Zhu Z, Tan L, Fu Y, Zhang W, Wang X, Xie D, Sun C. Origin of seed shattering in rice (Oryza sativa L.). Planta, 2007, 226: 11-20

[28] Konishi S, Izawa T, Lin S Y, Ebana K, Fukuta Y, Sasaki T, Yano M. An SNP caused loss of seed shattering during rice domestication. Science, 2006, 312: 1392-1396 
[29] Ji H S, Chu S H, Jiang W, Cho Y I, Hahn J H, Eun M Y, McCouch S R, Koh H J. Characterization and mapping of a shattering mutant in rice that corresponds to a block of domestication genes. Genetics, 2006, 173: 995-1005

[30] Luo J J, Hao W, Jin J, Gao J P, Lin H X. Fine mapping of Spr3, a locus for spreading panicle from African cultivated rice (Oryza glaberrima Steud.). Mol Plant, 2008, 1: 830-868

[31] Mao L, Begum D, Chuang H W, Budiman M A, Szymkowiak E J, Irish $\mathrm{E}$ E, Wing R A. Jointless is a MADS-box gene controlling tomato flower abscission zone development. Nature, 2000, 406: 910-913

[32] Szymkowiak E J, Irish E E. Interactions between jointless and wild-type tomato tissues during development of the pedicel abscission zone and the inflorescence meristem. Plant Cell, 1999, 11: $159-175$

[33] Brill E M, Watson J M. Ectopic expression of a Eucalyptus grandis SVP orthologue alters the flowering time of Arabidopsis thaliana. Funct Plant Biol, 2004, 31: 217-224

[34] Leseberg C H, Eissler C L, Wang X, Johns M A, Duvall M R, Mao L. Interaction study of MADS-domain proteins in tomato. $J$ Exp Bot, 2008, 59: 2253-2265

[35] Szymkowiak E J, Sussex I M. The internal meristem layer (L3) determines floral meristem size and carpel number in tomato periclinal chimeras. Plant Cell, 1992, 4: 1089-1100

[36] Yang T J, Lee S, Chang S B, Yu Y, de Jong H, Wing R A. In-depth sequence analysis of the tomato chromosome 12 centromeric region: Identification of a large CAA block and characterization of pericentromere retrotranposons. Chromosoma, 2005, 114: $103-117$

[37] Schumacher K, Schmitt T, Rossberg M, Schmitz G, Theres K. The lateral suppressor $(L S)$ gene of tomato encodes a new member of the VHIID protein family. Proc Natl Acad Sci USA, 1999, 96: $290-295$
[38] Jiang C Z, Lu F, Imsabai W, Meir S, Reid M S. Silencing polygalacturonase expression inhibits tomato petiole abscission. $J$ Exp Bot, 2008, 59: 973-979

[39] Brummell D A, Hall B D, Bennett A B. Antisense suppression of tomato endo-1,4-beta-glucanase Cel2 mRNA accumulation increases the force required to break fruit abscission zones but does not affect fruit softening. Plant Mol Biol, 1999, 40: 615-622

[40] Gonzalez-Bosch C, del Campillo E, Bennett A B. Immunodetection and characterization of tomato endo-beta-1,4-glucanase cel1 protein in flower abscission zones. Plant Physiol, 1997, 114: 1541-1546

[41] del Campillo E, Bennett A B. Pedicel breakstrength and cellulase gene expression during tomato flower abscission. Plant Physiol, 1996, 111: 813-820

[42] Guinn G, Brummett D L. Changes in abscisic acid and Indoleacetic acid before and after anthesis relative to changes in abscission rates of cotton fruiting forms. Plant Physiol, 1988, 87: 629-631

[43] Guinn G, Brummett D L. Changes in free and conjugated indole 3-acetic acid and abscisic acid in young cotton fruits and their abscission zones in relation to fruit retention during and after moisture stress. Plant Physiol, 1988, 86: 28-31

[44] Guinn G. Abscisic acid and cutout in cotton. Plant Physiol, 1985, 77: $16-20$

[45] Chen Q F, Yen C, Yang J L. Chromosome location of the gene for brittle rachis in the Tibetan weedrace of common wheat. Genet Resour Crop Evol, 1998, 45: 407-410

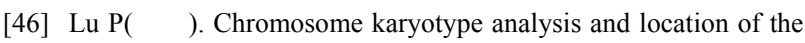
gene for brittle rachis in the Tibetan wheat. Tibetan J Agric Sci (西藏农业科技), 2000, 22(2): 23-27(in Chinese)

[47] Watanabe N, Fujii Y, Kato N, Ban T, Martinek P. Microsatellite mapping of the genes for brittle rachis on homoeologous group 3 chromosomes in tetraploid and hexaploid wheats. J Appl Genet, 2006, 47: 93-98 